This information is current as of April 25, 2023. 


\title{
Brain Structural and Vascular Anatomy Is Altered in Offspring of Pre-Eclamptic Pregnancies: A Pilot Study
}

(D) M.T. Rätsep, (D)A. Paolozza, (D)A.F. Hickman, (DB. Maser, (D).R. Kay, DS. Mohammad, (D). Pudwell, (D).N. Smith, (DD. Brien, (iD).W. Stroman, (DM.A. Adams, (D).N. Reynolds, (D)B.A. Croy, and DN.D. Forkert

\begin{abstract}
BACKGROUND AND PURPOSE: Pre-eclampsia is a serious clinical gestational disorder occurring in 3\%-5\% of all human pregnancies and characterized by endothelial dysfunction and vascular complications. Offspring born of pre-eclamptic pregnancies are reported to exhibit deficits in cognitive function, higher incidence of depression, and increased susceptibility to stroke. However, no brain imaging reports exist on these offspring. We aimed to assess brain structural and vascular anatomy in 7- to 10-year-old offspring of pre-eclamptic pregnancies compared with matched controls.
\end{abstract}

MATERIALS AND METHODS: Offspring of pre-eclamptic pregnancies and matched controls ( $n=10$ per group) were recruited from an established longitudinal cohort examining the effects of pre-eclampsia. Children underwent MR imaging to identify brain structural and vascular anatomic differences. Maternal plasma samples collected at birth were assayed for angiogenic factors by enzyme-linked immunosorbent assay.

RESULTS: Offspring of pre-eclamptic pregnancies exhibited enlarged brain regional volumes of the cerebellum, temporal lobe, brain stem, and right and left amygdalae. These offspring displayed reduced cerebral vessel radii in the occipital and parietal lobes. Enzyme-linked immunosorbent assay analysis revealed underexpression of the placental growth factor among the maternal plasma samples from women who experienced pre-eclampsia.

CONCLUSIONS: This study is the first to report brain structural and vascular anatomic alterations in the population of offspring of pre-eclamptic pregnancies. Brain structural alterations shared similarities with those seen in autism. Vascular alterations may have preceded these structural alterations. This pilot study requires further validation with a larger population to provide stronger estimates of brain structural and vascular outcomes among the offspring of pre-eclamptic pregnancies.

ABBREVIATIONS: MNI = Montreal Neurological Institute; PE = pre-eclampsia; PE-Fl = offspring of pre-eclamptic pregnancy; PGF = placental growth factor; sFLT-1 = soluble fms-like tyrosine kinase 1

$\mathbf{P}$ re-eclampsia (PE), a serious clinical gestational disorder, occurs in approximately $3 \%-5 \%$ of all pregnancies. ${ }^{1}$ The current clinical definition of $\mathrm{PE}$ involves new-onset hypertension $(>140 / 90 \mathrm{~mm} \mathrm{Hg}$ ) and at least 1 of the following: proteinuria ( $>300 \mathrm{mg} /$ day), thrombocytopenia (platelet level $<10^{5} / \mu \mathrm{L}$ ), re-

Received August 17, 2015; accepted after revision November 5.

From the Department of Biomedical and Molecular Sciences (M.T.R., A.F.H., B.M., V.R.K., S.M., G.N.S., J.N.R., B.A.C.) and Centre for Neuroscience Studies (A.P., D.B., P.W.S., M.A.A., J.N.R.), Queen's University, Kingston, Ontario, Canada; Department of Obstetrics and Gynecology (J.P., G.N.S.), Kingston General Hospital, Kingston, Ontario, Canada; and Department of Radiology and Hotchkiss Brain Institute (N.D.F.), University of Calgary, Calgary, Alberta, Canada.

This work was supported by the Harry Botterell Foundation for the Neurological Sciences Award from Kingston General Hospital Foundation, Canadian Institutes of Health Research; Heart and Stroke Foundation of Canada; and NeuroDevNet, which is funded by the Networks of Centers of Excellence, a federal government program to advance science and technology. B.A.C. is funded by the Canada Research Chairs program; M.T.R. is funded by a Frederick Banting and Charles Best Canada Graduate Scholarship from the Canadian Institutes of Health Research. nal insufficiency (serum creatinine level $>1.1 \mathrm{mg} / \mathrm{dL}$ ), impaired liver function (blood liver transaminases levels $2 \times$ normal), pulmonary edema, or cerebral or visual disturbances occurring after the 20th week of gestation. ${ }^{2} \mathrm{PE}$ is a severe acute disorder, requiring immediate action. Unfortunately, no effective treatments are available for $\mathrm{PE}$, and the only course of action is delivery of the fetus and placenta, often prematurely, which terminates maternal symptoms. Although many theories have been proposed for the origins of PE, specific causes and mechanisms of pathogenesis are poorly understood. A very popular theory is centered on deficient

\footnotetext{
Please address correspondence to Matthew T. Rätsep, PhD, Department of Biomedical and Molecular Sciences, Queen's University, Room 926, Botterell Hall, 18 Stuart St, Kingston, ON, Canada, K7L3N6; e-mail: m.ratsep@queensu.ca; @MTRatsep

-- Indicates open access to non-subscribers at www.ajnr.org

三 Indicates article with supplemental on-line table.

http://dx.doi.org/10.3174/ajnr.A4640
} 
uteroplacental angiogenesis and vessel remodeling, leading to increased vascular resistance, resulting in clinical signs. Many have posited systemic vascular endothelial dysfunction as the main factor underlying this deficient angiogenesis to explain the occurrence of the wide range of clinical indicators beyond hypertension. ${ }^{3,4}$

Given the strong association of PE with angiogenesis and vessel remodeling, numerous angiogenic biomarkers of $\mathrm{PE}$ have been proposed. ${ }^{5}$ Most important, deficient expression of placental growth factor (PGF) ${ }^{6,7}$ or overexpression of soluble endoglin ${ }^{7,8}$ or soluble fms-like tyrosine kinase 1 (sFLT-1) $)^{6,7}$ is strongly linked with the development of PE. In many cases, aberrant expression of these factors will precede clinical signs ${ }^{9}$ and may predict a more severe form of the disorder. ${ }^{10}$ Furthermore, women who experience a PE pregnancy with aberrant angiogenic factor expression will continue to exhibit an increased lifetime risk for cardiovascular disease. ${ }^{1,12}$ The lifetime risk outcomes are also suboptimal for the offspring of pre-eclamptic pregnancies (PE-F1s), who display an elevated risk of cardiovascular disease and stroke ${ }^{13,14}$ as well as impaired cognitive function ${ }^{15,16}$ and increased depressive signs. ${ }^{17}$ This finding raises the possibility that impairment in cerebral angiogenesis in PE-F1 fetuses leads to deficits in cognitive function while simultaneously increasing stroke risk. However, there appears to be no reported study on cerebrovascular anatomy in a PE-F1 population, to our knowledge.

Imaging of cerebral blood vessels can be achieved noninvasively with excellent quality, for example, by using TOF-MRA. With this method, high-resolution images of cerebral vasculature with a good blood-to-background contrast can be acquired without the administration of an exogenous contrast agent. In the current study, we have used high-resolution T1-weighted MR imaging to investigate regional brain volume alterations in combination with TOF-MRA for a quantitative analysis of the regional cerebral vasculature of children 7-10 years of age who were born of PE or uncomplicated pregnancies.

\section{MATERIALS AND METHODS Participants}

All experimental procedures were reviewed and approved by the Human Research Ethics Board at Queen's University and Kingston General Hospital. Participants were recruited from the PreEclampsia New Emerging Team study data base. ${ }^{18}$ Briefly, pregnant women were recruited to the Pre-Eclampsia New Emerging Team study at Kingston General Hospital between September 2003 and October 2009. Women were diagnosed with PE if blood pressure was $>140 / 90 \mathrm{~mm} \mathrm{Hg}$ and proteinuria level, $>300 \mathrm{mg} / 24$ hours or $\geq 2+$ on a repeat dipstick. Control women, with uncomplicated pregnancies, were enrolled and matched to test subjects for age, race, and parity. Women with a history of chronic hypertension, diabetes (including gestational diabetes), or renal disease were excluded. For this pilot study, women from the Pre-Eclampsia New Emerging Team data base were contacted between July 2014 and February 2015 to request participation of their child. PE-F1s 7-10 years of age ( $n=10 ; 5$ male, 5 female) were matched as closely as possible for age and sex to a child born of an uncomplicated pregnancy ( $n=10 ; 5$ male, 5 female). Following parental consent and participant assent, children underwent brain MR im- aging as described below. During the same session, the child's parent or guardian completed a questionnaire encompassing demographics, socioeconomic variables, pregnancy and birth information, the child's current health status, and family history variables. The accuracy of pregnancy and birth information was confirmed through access of medical records. Socioeconomic status was calculated according to Hollingshead Four Factor Index of Social Status. ${ }^{19}$ Participants received a Can $\$ 25$ gift card on completion of the testing session.

\section{MR Imaging}

All MR imaging was performed on a 3T Magnetom Trio MR imaging scanner (Siemens, Erlangen, Germany) without administration of contrast media or sedation. Among others, a highresolution TOF-MRA and a high-resolution anatomic T1weighted MPRAGE sequence were acquired for each participant. TOF-MRA was acquired by using 4 slabs, each consisting of 40 sections with a thickness of $0.5 \mathrm{~mm}$, a TE of $3.59 \mathrm{~ms}$, a TR of $20 \mathrm{~ms}$, a flip angle of $18^{\circ}$, and a spatial resolution of $0.26 \times 0.26 \mathrm{~mm}^{2}$. Because TOF-MRA imaging provides a good blood-to-background contrast of arteries but little anatomic information, highresolution T1-weighted MPRAGE images were used as the basis for the regional vessel and brain volume analysis. The MPRAGE sequence was acquired in a single 160-section slab, by using a TE of $3.45 \mathrm{~ms}$, a TR of $2180 \mathrm{~ms}$, a flip angle of $15^{\circ}$, and an isotropic spatial resolution of $1.0 \mathrm{~mm}^{3}$.

\section{Data Analyses}

For the vessel analyses, the cerebrovascular system was segmented in each TOF-MRA dataset by using an advanced multistep segmentation framework. ${ }^{20-22}$ On the basis of the resulting vessel segmentation, the $3 \mathrm{D}$ vessel centerline representation and the corresponding vessel radius for each voxel of the vessel centerline were then calculated by using the method described by Forkert et al (Figure). ${ }^{23}$ Due to different FOVs of the TOF-MRA acquisition and to enable an analysis of the regional vascular alterations, parcellation of each brain into anatomic substructures was required. First, the well-established Montreal Neurological Institute (MNI) adult brain atlas ${ }^{24}$ was registered to the MNI pediatric atlas (7-11 years of age $)^{25}$ by optimizing the nonlinear transformation $\varphi_{\mathrm{A}}$. Next, the MNI pediatric atlas was registered to each MPRAGE dataset by optimizing the nonlinear transformation $\varphi_{P}$. Finally, each MPRAGE dataset was registered to the corresponding TOFMRA dataset of the same participant by optimizing the rigid transformation $\varphi_{\mathrm{M}}$. All registrations were performed by using the NiftyReg software (http://sourceforge.net/projects/niftyreg/) ${ }^{26}$ and were visually checked for quality control. A full intracranial brain volume segmentation, the MNI brain regions, and the Harvard-Oxford subcortical brain regions, as defined in the MNI adult brain atlas, were then transformed to each MPRAGE dataset by concatenating the 2 nonlinear transformations $\left(\varphi_{\mathrm{A}^{\circ}} \varphi_{\mathrm{P}}\right)$ as well as to the TOF-MRA dataset by concatenating the 2 nonlinear and the rigid transformations $\left(\varphi_{\mathrm{A}^{\circ}} \varphi_{\mathrm{P}^{\circ}} \varphi_{\mathrm{M}}\right)$ by using a nearest-neighbor interpolation.

After registration, the subject-specific full intracranial brain volume and the volume of each of the MNI and Harvard-Oxford subcortical brain regions, as defined by a probabilistic threshold 


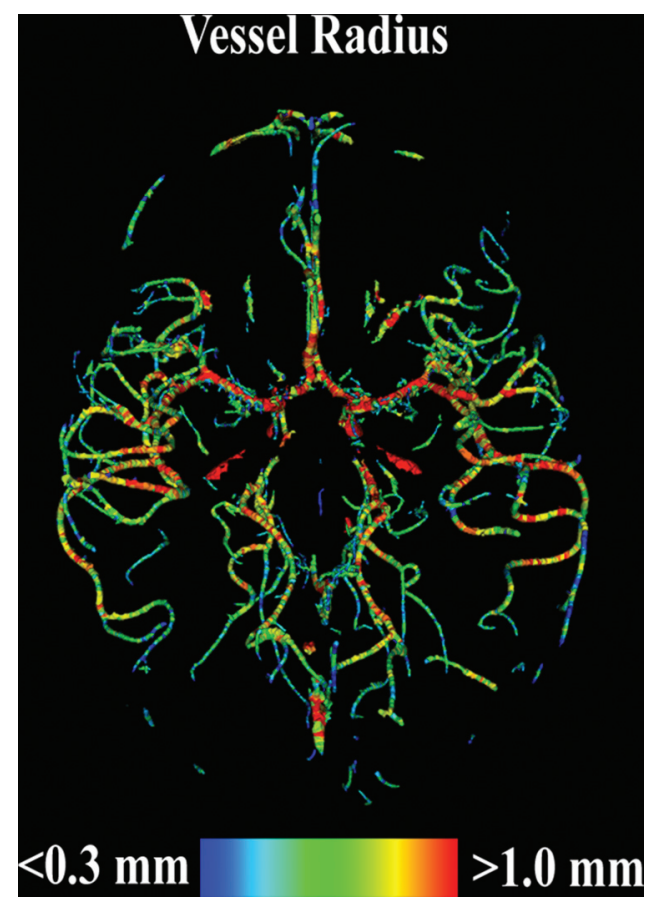

FIGURE. Visual 3D representation of the vessel radii calculated for a segmented TOF-MRA dataset of a single female, 8-year-old PE-Fl participant who was born at 34 weeks of gestation.

of $50 \%$, were extracted in T1 space. The full intracranial volume was used to calculate normalized regional brain volumes to account for different head geometries. Normalized regional brain volumes are expressed as a percentage of the full intracranial volume. The MNI brain regions extracted and quantified were the cerebellum, frontal lobe, occipital lobe, parietal lobe, and temporal lobe. The Harvard-Oxford brain regions extracted and quantified were the amygdalae, brain stem, caudate, cerebral cortex, cerebral white matter, lateral ventricles, pallidum, putamen, and thalamus. The volume of the Harvard-Oxford subcortical brain regions was calculated separately for the left and right hemispheres, except for the brain stem.

For the regional vessel analysis, the mean vessel radius and the vascular attenuation were calculated only for the larger MNI and Harvard-Oxford brain regions because smaller regions are too susceptible to noise artifacts. The MNI brain regions used for vessel analysis were the cerebellum, frontal lobe, occipital lobe, parietal lobe, and temporal lobe. Harvard-Oxford brain regions used for vessel analysis were the cerebral white matter and cerebral cortex. Vascular attenuation within each region was calculated by dividing the total volume of segmented vascular structures within a given region by the total volume of brain tissue in that same region. This value was then multiplied by 100 to be expressed as a percentage.

\section{Enzyme-Linked Immunosorbent Assays}

Pregnant women recruited to the Pre-Eclampsia New Emerging Team study were asked to give peripartum blood samples. Of the participants used in this study, 4 women with PE and 8 control women consented to blood sample donation. Samples were collected in heparin and were stored at $-80^{\circ} \mathrm{C}$ until used for analysis. Blood samples were assayed by enzyme-linked immunosorbent assays for PGF, sFLT-1, and soluble endoglin by using purchased kits (Quantikine; R\&D Systems, Minneapolis, Minnesota); enzyme-linked immunosorbent assays were performed according to the manufacturer's instructions. PE and control samples were diluted 1:1 for PGF, 1:25 for sFLT-1, and 1:4 for soluble endoglin.

\section{Statistics}

Equality of variances of continuous variables between groups was assessed by the $F$ test. Differences in continuous variables between groups were analyzed by 2-tailed unpaired $t$ tests for demographic and plasma protein data using the Satterthwaite approximation for unequal variances when appropriate. Differences in categoric variables between groups were analyzed by the $\chi^{2}$ test or Fisher exact test when expected counts were $<5$. Differences in parameters extracted from MR imaging between groups were analyzed by multivariate analysis of covariance with consideration to age, height, and weight of the participants as covariates. The multivariate effect of each independent variable was assessed by the Wilks $\lambda$, which is a measure of the proportion of variance of the dependent variables that is accounted for by the independent variable. Adjusted means were calculated for each parameter on the basis of the linear models produced in the MANCOVA analysis. Pair-wise comparisons were corrected for multiple comparisons by the Bonferroni method. All data are expressed as mean \pm standard error, unless stated otherwise. Differences between groups were considered statistically significant at a $P$ value $<.05$. Statistical analyses were conducted by using SAS, Version 9.3 (SAS Institute, Cary, North Carolina) and SPSS, Version 22 (IBM, Armonk, New York).

\section{RESULTS}

The characteristics of the study samples are displayed in Table 1. The PE-F1 and control groups differed significantly in birth weight (control: $3419.9 \pm 361.2$ g versus PE: $2665.2 \pm 788.7 \mathrm{~g}$, $P=.013)$. All other characteristics, including gestational age at birth, were not significantly different between the PE-F1 and control groups.

The MANCOVA analysis showed no overall effect of PE status $(\lambda=0.058, P=.649)$. Similarly, none of the covariates displayed a significant overall effect on the extracted parameters (age: $\lambda=$ $0.024, P=.448$; height: $\lambda=0.020, P=.416$; weight: $\lambda=0.041$, $P=.566$ ). Post hoc analyses did reveal significant differences among pair-wise comparisons of individual extracted parameters. The MPRAGE imaging revealed no significant difference in total intracranial volume between the control and PE groups (control: $1431.7 \pm 33.6 \mathrm{~mL}$ versus PE: $1495.5 \pm 33.6 \mathrm{~mL}, P=.201$; Table $2)$. The PE group displayed statistically significantly larger adjusted mean normalized volumes in 5 brain regions: cerebellum (control: $8.72 \pm 0.21 \%$ versus PE: $9.58 \pm 0.21 \%, P=.010$; Table 2), temporal lobe (control: $8.98 \pm 0.15 \%$ versus $\mathrm{PE}$ : $9.65 \pm$ $0.15 \%, P=.007$; Table 2), left amygdala (control: $0.09 \pm 0.003 \%$ versus $\mathrm{PE}: 0.10 \pm 0.003 \%, P=.023$; Table 2$)$, right amygdala (control: $0.11 \pm 0.002 \%$ versus $\mathrm{PE}: 0.12 \pm 0.002 \%, P=.012$; Table 2), and brain stem (control: $1.27 \pm 0.04 \%$ versus PE: $1.40 \pm$ $0.04 \%, P=.015$; Table 2). None of the other 21 brain regions analyzed displayed statistically significant differences (Table 2).

The analysis of the TOF-MRA datasets revealed significant 
Table 1: Study population characteristics

\begin{tabular}{|c|c|c|c|}
\hline Characteristics at Testing & Control $(n=10)$ & $\operatorname{PE}(n=10)$ & $P$ Value $^{\mathrm{b}}$ \\
\hline Age (yr) (mean) (SD) & $9.79(0.89)$ & $9.66(1.07)$ & $.769^{c}$ \\
\hline Male sex (No.) (\%) & $5(50.0)$ & $5(50.0)$ & $1.000^{\mathrm{d}}$ \\
\hline Height (m) (mean) (SD) & $1.37(0.09)$ & $1.39(0.14)$ & $.644^{c}$ \\
\hline Weight (kg) (mean) (SD) & $30.12(5.68)$ & $30.66(9.19)$ & $.875^{c}$ \\
\hline BMI $\left(\mathrm{kg} / \mathrm{m}^{2}\right)$ (mean) (SD) & $16.08(1.93)$ & $16.17(5.45)$ & $.962^{\mathrm{c}}$ \\
\hline Caucasian (No.) (\%) & $10(100)$ & $10(100)$ & $1.000^{d}$ \\
\hline Household SES ${ }^{a}$ (mean) (SD) & $49.15(9.07)$ & $51.10(9.10)$ & $.637^{c}$ \\
\hline Psychiatric disorder (No.) (\%) & & & $.303^{d}$ \\
\hline Anxiety & $0(0)$ & $2(20)$ & \\
\hline Depression & $0(0)$ & $1(10)$ & \\
\hline ADHD & $1(10)$ & $2(20)$ & \\
\hline \multicolumn{4}{|l|}{ Characteristics at birth } \\
\hline Gestational age (wks) (mean) (SD) & $39.47(1.38)$ & $37.16(3.34)$ & $.055^{c}$ \\
\hline Birth weight (g) (mean) (SD) & $3419.9(361.2)$ & $2665.2(788.7)$ & $.013^{c}$ \\
\hline Length $(\mathrm{cm})$ (mean) (SD) & $51.38(3.39)$ & $48.50(4.36)$ & $.146^{\mathrm{C}}$ \\
\hline Head circumference $(\mathrm{cm})$ (mean) (SD) & $34.31(0.59)$ & $32.65(3.01)$ & $.120^{c}$ \\
\hline Abdominal girth (cm) (mean) (SD) & $32.69(2.22)$ & $29.81(3.10)$ & $.051^{\mathrm{C}}$ \\
\hline Delivery type (No.) (\%) & & & $.070^{\mathrm{d}}$ \\
\hline Vaginal & $8(80)$ & $3(30)$ & \\
\hline Cesarean birth & $2(20)$ & $7(70)$ & \\
\hline Maternal height (m) (mean) (SD) & $1.64(0.07)$ & $1.65(0.09)$ & $.716^{\mathrm{c}}$ \\
\hline Maternal weight $(\mathrm{kg})$ (mean) (SD) & $80.44(14.31)$ & $84.80(16.96)$ & $.556^{\mathrm{c}}$ \\
\hline Maternal BMI $\left(\mathrm{kg} / \mathrm{m}^{2}\right)$ (mean) (SD) & $30.06(6.26)$ & $30.99(5.41)$ & $.735^{\mathrm{c}}$ \\
\hline Maternal age (yr) (mean) (SD) & $32.27(4.58)$ & $30.59(3.23)$ & $.355^{c}$ \\
\hline
\end{tabular}

Note:-SES indicates socioeconomic status; ADHD, attention deficit/hyperactivity disorder; BMI, body mass index.

${ }^{a}$ Calculated according to Hollingshead Four Factor Index of Social Status.

b Bonferroni-corrected.

c Student $t$ test.

${ }^{d}$ Fisher exact test.

Table 2: Adjusted mean brain regional volumes (percentage of full intracranial volume) ${ }^{a}$

\begin{tabular}{|c|c|c|c|}
\hline Brain Region & $\begin{array}{l}\text { Control }(n=10) \\
\text { (mean) (SE) }\end{array}$ & $\begin{array}{l}\mathrm{PE}(n=10) \\
\text { (mean) (SE) }\end{array}$ & $P$ Value $^{\mathrm{d}}$ \\
\hline Full intracranial volume $(\mathrm{mL})$ & $1431.7(33.6)$ & $1495.5(33.6)$ & .201 \\
\hline Cerebellum $^{\mathrm{b}}$ & $8.72(0.21)$ & $9.58(0.21)$ & .010 \\
\hline Frontal lobe ${ }^{b}$ & $16.62(0.39)$ & $17.08(0.39)$ & .422 \\
\hline Occipital lobe & $6.25(0.18)$ & $6.58(0.18)$ & .213 \\
\hline Parietal lobe $e^{b}$ & $9.41(0.26)$ & $9.75(0.26)$ & .364 \\
\hline Temporal lobe ${ }^{b}$ & $8.98(0.15)$ & $9.65(0.15)$ & .007 \\
\hline Left accumbens ${ }^{c}$ & $0.03(0.001)$ & $0.03(0.001)$ & .993 \\
\hline Right accumbens ${ }^{c}$ & $0.03(0.001)$ & $0.03(0.001)$ & .986 \\
\hline Left amygdalac & $0.09(0.003)$ & $0.10(0.003)$ & .023 \\
\hline Right amygdalac & $0.11(0.002)$ & $0.12(0.002)$ & .012 \\
\hline Brain stem ${ }^{c}$ & $1.27(0.04)$ & $1.40(0.04)$ & .015 \\
\hline Left caudate ${ }^{c}$ & $0.20(0.01)$ & $0.21(0.01)$ & .843 \\
\hline Right caudate ${ }^{c}$ & $0.20(0.01)$ & $0.21(0.01)$ & .490 \\
\hline Left cerebral cortex ${ }^{c}$ & $23.25(0.41)$ & $24.39(0.41)$ & .071 \\
\hline Right cerebral cortex ${ }^{c}$ & $24.42(0.46)$ & $25.39(0.46)$ & .158 \\
\hline Left cerebral white matter ${ }^{c}$ & $13.42(0.37)$ & $13.96(0.37)$ & .320 \\
\hline Right cerebral white matter & $12.17(0.33)$ & $12.85(0.33)$ & .168 \\
\hline Left hippocampus ${ }^{c}$ & $0.21(0.01)$ & $0.23(0.01)$ & .166 \\
\hline Right hippocampus ${ }^{c}$ & $0.22(0.01)$ & $0.23(0.01)$ & .247 \\
\hline Left lateral ventricle $e^{c}$ & $0.34(0.07)$ & $0.36(0.07)$ & .873 \\
\hline Right lateral ventricle $e^{c}$ & $0.31(0.05)$ & $0.32(0.05)$ & .941 \\
\hline Left pallidum ${ }^{c}$ & $0.11(0.004)$ & $0.12(0.004)$ & .327 \\
\hline Right pallidum ${ }^{c}$ & $0.12(0.004)$ & $0.12(0.004)$ & .257 \\
\hline Left putamen ${ }^{c}$ & $0.31(0.01)$ & $0.32(0.01)$ & .446 \\
\hline Right putamen ${ }^{c}$ & $0.34(0.01)$ & $0.34(0.01)$ & .594 \\
\hline Left thalamus ${ }^{c}$ & $0.36(0.01)$ & $0.38(0.01)$ & .335 \\
\hline Right thalamus ${ }^{c}$ & $0.41(0.01)$ & $0.42(0.01)$ & .396 \\
\hline
\end{tabular}

Note:-SE indicates standard error.

${ }^{a}$ All means were adjusted for age (9.72 yr), height (1.38 m), and weight. (30.39 kg).

b Derived from the Montreal Neurological Institute brain atlas.

cDerived from the Harvard-Oxford subcortical atlas.

d Bonferroni-corrected. differences in 2 regions: the occipital lobe and parietal lobe. In the occipital lobe, the mean vessel radius was significantly smaller in the PE group (control: $0.50 \pm 0.01 \mathrm{~mm}$ versus PE: $0.45 \pm 0.01$ $\mathrm{mm}, P=.004$; Table 3). Similarly, in the parietal lobe, the mean vessel radius was significantly smaller in the PE group (control: $0.55 \pm 0.01 \mathrm{~mm}$ versus PE: $0.52 \pm 0.01 \mathrm{~mm}, P=.025$; Table 3 ). There were no significant differences in vessel radius between the PE and control groups for any of the other brain regions analyzed (Table 3). Additionally, there were no significant differences in vascular attenuation between the PE and control groups for any of the 7 brain regions analyzed (On-line Table).

Analysis of maternal plasma samples by enzyme-linked immunosorbent assay revealed that $\mathrm{PE}$ samples expressed significantly lower levels of PGF (control: $221.0 \pm 46.6 \mathrm{pg} / \mathrm{mL}$ versus $\mathrm{PE}$ : $37.2 \pm 21.5 \mathrm{pg} / \mathrm{mL}, P=.024)$. PE samples did not display statistically significant different concentrations of sFLT-1 (control: 12,272 $\pm 3302 \mathrm{pg} / \mathrm{mL}$ versus PE: $23,596 \pm 13,630 \mathrm{pg} / \mathrm{mL}, P=.479$ ) or soluble endoglin (control: $13.34 \pm 3.40$ ng/mL versus $P E: ~ 40.43 \pm 21.10 \mathrm{ng} / \mathrm{mL}$, $P=.294)$ compared with controls.

\section{DISCUSSION}

Although numerous reports exist regarding cognitive function, ${ }^{15}$ risk of depression, ${ }^{17}$ or stroke ${ }^{14}$ among PE-F1s, our study aimed to link these outcomes to potential underlying defects in structural development. Prematurity has long been known to adversely affect brain growth and development and is linked to increased likelihood of variation of the circle of Willis. ${ }^{27}$ Although the effects of prematurity cannot be completely ruled out, the PE-F1s examined in this study were not born significantly earlier in gestation than the cohort-matched controls. Additionally, children of each group were not significantly different with respect to height or weight at the time of testing. Thus, longterm effects of PE on the growth trajectory are not apparent. Thus, the effects seen in the PE-F1s in this study can be attributed to the experience of gestation in a PE pregnancy. This would be consistent with previous findings of 
Table 3: Adjusted mean brain regional vascular diameters $(\mathbf{m m})^{\mathrm{a}}$

\begin{tabular}{lccc}
\hline \multicolumn{1}{c}{ Brain Region } & $\begin{array}{c}\text { Control }(\boldsymbol{n}=10) \\
(\text { mean) }(\mathrm{SE})\end{array}$ & $\begin{array}{c}\text { PE }(\boldsymbol{n}=10) \\
(\text { mean) }(\mathrm{SE})\end{array}$ & $\boldsymbol{P ~ V a l u e ~}^{\mathrm{d}}$ \\
\hline Cerebellum $^{\mathrm{b}}$ & $0.56(0.01)$ & $0.55(0.01)$ & .417 \\
Frontal lobe $^{\mathrm{b}}$ & $0.54(0.01)$ & $0.52(0.01)$ & .112 \\
Occipital lobe $^{\mathrm{b}}$ & $0.50(0.01)$ & $0.45(0.01)$ & .004 \\
Parietal lobe $^{\mathrm{b}}$ & $0.55(0.01)$ & $0.52(0.01)$ & .025 \\
Temporal lobe $^{\mathrm{b}}$ & $0.63(0.01)$ & $0.60(0.01)$ & .128 \\
Cerebral cortex $^{\mathrm{c}}$ & $0.57(0.01)$ & $0.55(0.01)$ & .085 \\
Cerebral white matter $^{\mathrm{c}}$ & $0.44(0.02)$ & $0.42(0.02)$ & .454 \\
\hline
\end{tabular}

${ }^{a}$ All means were adjusted for age $(9.72 \mathrm{yr})$, height $(1.38 \mathrm{~m})$, and weight $(30.39 \mathrm{~kg})$.

${ }^{\mathrm{b}}$ Derived from the Montreal Neurological Institute brain atlas.

Derived from the Harvard-Oxford subcortical atlas.

d Bonferroni-corrected.

impairment among PE-F1s compared with offspring born of pregnancies complicated by gestational hypertension. ${ }^{15,16}$

As expected, the maternal plasma samples displayed an aberrant expression pattern of an angiogenic factor (ie, underexpression of the proangiogenic PGF). Unexpectedly, however, the PEF1s displayed larger volumes in 5 brain regions (cerebellum, temporal lobe, brain stem, left and right amygdalae) but smaller vessel radii in the occipital and parietal lobes. In line with this finding, all other regions examined also displayed a nonsignificant trend for smaller vessel radii for PE-F1s compared with controls. This finding suggests that the aberrant maternal angiogenic signaling milieu common among PE pregnancies adversely affects brain vascular growth in the offspring. The described vessel measurements are mostly related to vessels in the sulci that are responsible for the blood supply of the brain tissue. Although these arteries are, strictly speaking, outside the brain regions used, they are still covered by the brain atlas regions because a probabilistic threshold of $50 \%$ was used for definition of the binary brain regions. Nevertheless, the reduction in brain vessel radii among PE-F1s does not appear to affect brain growth. This finding raises the question of how PE gestation leads to deficits in cognitive function and increased susceptibility to stroke in the offspring. The answer may lie in the disrupted general architecture of the brain, potentially causing imbalances in signaling between adjacent regions. This is a phenomenon seen in autism spectrum disorder and attention deficit/hyperactivity disorder, in which the growth trajectory of the brain is disrupted, particularly in early life. ${ }^{28,29}$

The regional brain volumetric alterations seen in this study may further shed light on the specific cognitive functional profile of PE-F1s. Enlarged left and right amygdalae are also commonly seen in autism spectrum disorder ${ }^{30}$ and are associated with an increased incidence of temporal lobe epilepsy. ${ }^{31,32}$ Most interesting, the temporal lobe was also enlarged in PE-F1s compared with controls. A commonly prescribed antiepileptic, valproic acid, can also induce hypertrophy of the amygdalae, leading to autistic-like behaviors in a rat model..$^{33}$ Additionally, early life stress has been shown to have an effect on amygdala hypertrophy in a macaque model, acting through serotonin signaling. ${ }^{34}$ This is mirrored in a study of children, showing that enlarged amygdalae are associated with increased anxiety. ${ }^{35}$ Cerebellar and brain stem enlargements are common among small-for-gestational-age neonates at term. ${ }^{36}$ These enlargements correlated significantly with cluster scores from the Neonatal Behavioral Assessment Scale. ${ }^{36}$ Additionally, children born very preterm display delays in cortical thinning compared with children born at term. ${ }^{37}$ Thus, the brain anomalies observed among PE-F1 in the present study may be caused by a combined effect of a PE gestation in addition to prematurity and fetal growth restriction. Further study will be necessary to separate the effects of each factor on brain development in these children.

Our data also agree well with studies of animal models of PE. In a mouse model of $\mathrm{PE}$ induced by pregnancy-specific lentiviral overexpression of sFLT-1, offspring displayed structural alterations in numerous brain regions. ${ }^{38}$ In this case, many of the structural alterations were enlargements, with patterns of alterations differing between sexes. Furthermore, this model induced impairments in vestibular function, balance, and coordination in the offspring. ${ }^{39}$ In both cases, maternal treatment with pravastatin prevented the structural and functional impairments seen in the offspring. Furthermore, brain developmental and functional impairments were displayed in the offspring of a rat model of $\mathrm{PE}$ induced by maternal treatment with $\mathrm{N} \omega$-nitro-L-arginine methyl ester, a potent nitric oxide synthase inhibitor. ${ }^{40}$ The authors found that the $\mathrm{N} \omega$-nitro-L-arginine methyl ester-treated rat offspring displayed impaired neurogenesis but increased gliogenesis, which compensated for the reduction in neurogenesis to normalize brain weight. Therefore, although brain structural anatomy was essentially normal in the offspring of this model, the treatment led to functional deficits in spatial learning and memory. ${ }^{40}$

Our pilot study provides fresh insight into the well-documented cognitive functional outcomes among PE-F1s. By examining children rather than adults, though somewhat more challenging, our study results are less susceptible to confounding from long-term environmental exposures that may also deviate cognitive functioning. Additionally, we are able to detect anomalies in specific brain regions relatively early in life, when interventional strategies may still provide very profound beneficial long-term effects. Although sample size and range in childhood ages are limited, our findings are consistent with numerous previously published studies that have consistently shown impaired cognitive functions among PE-F1s. ${ }^{15}$ Through this study, we have provided a foundation on which future studies can build. The focus within our group will be to increase participant recruitment for this study to provide more accurate estimates of how brain vascular and structural alterations may lead to functional deficits. Within this context, the results of this study need to be considered exploratory at this stage because of the rather small sample size. On the basis of the clear differences found in the vascular anatomy, it appears most fruitful to investigate the cerebrovascular system in more detail (eg, by investigating the branching index of vessels in different brain regions or even by developing vascular network analysis methods). ${ }^{41}$

\section{CONCLUSIONS}

We have shown that PE-F1 as children display significant enlargement of the cerebellum, brain stem, temporal lobe, and right and left amygdalae. Additionally, PE-F1s display significantly reduced vessel radii in the occipital and parietal lobes. These structural and vascular anomalies may underlie the cognitive deficits reported in 
PE-F1 populations and may contribute to an elevated lifetime risk of depression and stroke.

\section{ACKNOWLEDGMENTS}

We thank the participants and their families for taking part in the study. We thank Mrs Aysha Tayab, Queen's University, for assistance in participant recruitment and acquiring questionnaire data, and Ms Janelle Zhan, Queen's University, for assistance in acquiring questionnaire data.

Disclosures: Matthew T. Rätsep—RELATED: Grant: Kingston General Hospital Foundation; Canadian Institutes of Health Research. * Vanessa R. Kay—RELATED: Grant: Grant funding for the lab is disclosed on the Principal Investigator's disclosure form*; Support for Travel to Meetings for the Study or Other Purposes: Travel Award from the Canadian Institutes of Health Research to attend the Gordon Research Conference on Angiogenesis (August 2015); Other: Canadian Institutes of Health Research,* Comments: MD/PhD program funding support to the institution; the program provides me (a student) with stipend. Queen's University also provides stipend support. Michael A. Adams—RELATED: Grant: Canadian Institutes of Health Research.* James N. Reynolds—RELATED: Grant: NeuroDevNet, * Comments: NeuroDevNet is funded by the Networks of Centers of Excellence, a program of the Canadian federal government to advance science and technology; UNRELATED: Grants/Grants Pending: NeuroDevNet, ${ }^{*}$ Comments: NeuroDevNet is funded by the Networks of Centers of Excellence, a program of the Canadian federal government to advance science and technology. Barbara Anne Croy-RELATED: Grant: Harry Botterell Foundation for the Neurological Sciences Award, ${ }^{*}$ Comments: Internal Institutional award administered through Queen's University, Kingston, Ontario, for the Kingston General Hospital Research Foundation. The amount of the award was Can\$10,000. I was the Principal Investigator on the 1-year award made June 1, 2014. These funds covered the MRI user fees for the studies reported in the article; UNRELATED: Employment: I am an employee of Queen's University, Comments: The funds awarded were in an internal university competition, and a proposal was written and ranked by others in a peer review for this and all other Kingston General Hospital Foundation Awards; Grants/Grants Pending: Garfield Kelly and Harry Botterell Foundation for the Neurological Sciences, ${ }^{*}$ Comments: I received internal competitive funds of Can $\$ 10,000$ from my university for June 1, 2015 from the Garfield Kelly Award (Can\$2500) and the Botterell Foundation (Can\$7500). The former enabled the enzyme-linked immunosorbent assay studies reported in the article. The Botterell funds will permit us to access a second pregnancy cohort, the OaK (Ottawa and Kingston cohort), and support MRI user fees to study 4 more pairs of children. *Money paid to the institution.

\section{REFERENCES}

1. Roberts CL, Ford JB, Algert CS, et al. Population-based trends in pregnancy hypertension and pre-eclampsia: an international comparative study. BMJ Open 2011;1:e000101 CrossRef Medline

2. American College of Obstetricians and Gynecologists, Task Force on Hypertension in Pregnancy. Hypertension in pregnancy: report of the American College of Obstetricians and Gynecologists' Task Force on Hypertension in Pregnancy. Obstet Gynecol 2013;122: 1122-31 CrossRef Medline

3. Brennan LJ, Morton JS, Davidge ST. Vascular dysfunction in preeclampsia. Microcirculation 2014;21:4-14 CrossRef Medline

4. Turner RJ, Bloemenkamp KW, Penning ME, et al. From glomerular endothelium to podocyte pathobiology in preeclampsia: a paradigm shift. Cur Hypertens Rep 2015;17:54 CrossRef Medline

5. Staff AC, Benton SJ, von Dadelszen $P$, et al. Redefining preeclampsia using placenta-derived biomarkers. Hypertension 2013;61:932-42 CrossRef Medline

6. Levine RJ, Maynard SE, Qian C, et al. Circulating angiogenic factors and the risk of preeclampsia. N Engl J Med 2004;350:672-83 CrossRef Medline

7. Powers RW, Jeyabalan A, Clifton RG, et al; Eunice Kennedy Shriver National Institute of Child Health Human Development MaternalFetal Medicine Units Network. Soluble fms-like tyrosine kinase 1 (sFlt1), endoglin and placental growth factor (PlGF) in preeclampsia among high risk pregnancies. PLoS One 2010;5:e13263 CrossRef Medline

8. De Vivo A, Baviera G, Giordano D, et al. Endoglin, PlGF and sFlt-1 as markers for predicting pre-eclampsia. Acta Obstet Gynecol Scand 2008;87:837-42 CrossRef Medline

9. Thadhani R, Mutter WP, Wolf M, et al. First trimester placental growth factor and soluble fms-like tyrosine kinase $\mathbf{1}$ and risk for preeclampsia. J Clin Endocrinol Metab 2004;89:770-75 CrossRef Medline

10. Powers RW, Roberts JM, Plymire DA, et al. Low placental growth factor across pregnancy identifies a subset of women with preterm preeclampsia: type 1 versus type 2 preeclampsia? Hypertension 2012; 60:239-46 CrossRef Medline

11. Melchiorre K, Sutherland GR, Liberati M, et al. Preeclampsia is associated with persistent postpartum cardiovascular impairment. Hypertension 2011;58:709-15 CrossRef Medline

12. Östlund E, Al-Nashi M, Hamad RR, et al. Normalized endothelial function but sustained cardiovascular risk profile 11 years following a pregnancy complicated by preeclampsia. Hypertens Res 2013; 36:1081-87 CrossRef Medline

13. Davis EF, Lazdam M, Lewandowski AJ, et al. Cardiovascular risk factors in children and young adults born to preeclamptic pregnancies: a systematic review. Pediatrics 2012;129:e1552-61 CrossRef Medline

14. Kajantie E, Eriksson JG, Osmond C, et al. Pre-eclampsia is associated with increased risk of stroke in the adult offspring: the Helsinki birth cohort study. Stroke 2009;40:1176-80 CrossRef Medline

15. Tuovinen S, Eriksson JG, Kajantie E, et al. Maternal hypertensive pregnancy disorders and cognitive functioning of the offspring: a systematic review. J Am Soc Hypertens 2014;8:832-47.e1 CrossRef Medline

16. Tuovinen S, Räikkönen K, Pesonen AK, et al. Hypertensive disorders in pregnancy and risk of severe mental disorders in the offspring in adulthood: the Helsinki Birth Cohort Study. J Psychiatry Res 2012; 46:303-10 CrossRef Medline

17. Tuovinen S, Räikkönen K, Kajantie E, et al. Depressive symptoms in adulthood and intrauterine exposure to pre-eclampsia: the Helsinki Birth Cohort Study. BJOG 2010;117:1236-42 CrossRef Medline

18. Smith GN, Walker MC, Liu A, et al. A history of preeclampsia identifies women who have underlying cardiovascular risk factors. $A m J$ Obstet Gynecol 2009;200:58 e1-8 CrossRef Medline

19. Hollingshead AB. Four Factor Index of Social Status. Yale J Sociology 2011;8:21-51

20. Forkert ND, Schmidt-Richberg A, Fiehler J, et al. Fuzzy-based vascular structure enhancement in time-of-flight MRA images for improved segmentation. Meth Inf Med 2011;50:74-83 CrossRef Medline

21. Forkert ND, Schmidt-Richberg A, Fiehler J, et al. 3D cerebrovascular segmentation combining fuzzy vessel enhancement and level-sets with anisotropic energy weights. Magn Reson Imaging 2013;31: 262-71 CrossRef Medline

22. Forkert ND, Schmidt-Richberg A, Fiehler J, et al. Automatic correction of gaps in cerebrovascular segmentations extracted from 3D time-of-flight MRA datasets. Meth InfMed 2012;51:415-22 CrossRef Medline

23. Forkert ND, Fiehler J, Suniaga S, et al. A statistical cerebroarterial atlas derived from 700 MRA datasets. Meth Inf Med 2013;52:467-74 CrossRef Medline

24. Mazziotta J, Toga A, Evans A, et al. A probabilistic atlas and reference system for the human brain: International Consortium for Brain Mapping (ICBM). Philos Trans R Soc Lond B Biol Sci 2001;356: 1293-322 CrossRef Medline

25. Fonov V, Evans AC, Botteron K, et al; Brain Development Cooperative Group. Unbiased average age-appropriate atlases for pediatric studies. Neuroimage 2011;54:313-27 CrossRef Medline

26. Modat M, McClelland J, Ourselin S. Lung registration using the NiftyReg package. In: Proceedings of the International Conference on Medical Image Computing and Computer Assisted Intervention, Beijing, China. September 20-24, 2010

27. van Kooij BJ, Hendrikse J, Benders MJ, et al. Anatomy of the circle 
of Willis and blood flow in the brain-feeding vasculature in prematurely born infants. Neonatology 2010;97:235-41 CrossRef Medline

28. Courchesne E, Karns CM, Davis HR, et al. Unusual brain growth patterns in early life in patients with autistic disorder: an MRI study. Neurology 2001;57:245-54 CrossRef Medline

29. Konrad K, Eickhoff SB. Is the ADHD brain wired differently? A review on structural and functional connectivity in attention deficit hyperactivity disorder. Hum Brain Mapp 2010;31:904-16 CrossRef Medline

30. Nordahl CW, Scholz R, Yang X, et al. Increased rate of amygdala growth in children aged 2 to 4 years with autism spectrum disorders: a longitudinal study. Arch Gen Psychiatry 2012;69:53-61 CrossRef Medline

31. Sone D, Ito K, Taniguchi G, et al. Evaluation of amygdala pathology using (11)C-methionine positron emission tomography/computed tomography in patients with temporal lobe epilepsy and amygdala enlargement. Epilepsy Res 2015;112:114-21 CrossRef Medline

32. Lv RJ, Sun ZR, Cui T, et al. Temporal lobe epilepsy with amygdala enlargement: a subtype of temporal lobe epilepsy. BMC Neurol 2014;14:194 CrossRef Medline

33. Olde Loohuis NF, Kole K, Glennon JC, et al. Elevated microRNA$181 \mathrm{c}$ and microRNA-30d levels in the enlarged amygdala of the valproic acid rat model of autism. Neurobiol Dis 2015;80:42-53 CrossRef Medline

34. Coplan JD, Fathy HM, Jackowski AP, et al. Early life stress and macaque amygdala hypertrophy: preliminary evidence for a role for the serotonin transporter gene. Front Behav Neurosci 2014;8:342 CrossRef Medline

35. Qin S, Young CB, Duan X, et al. Amygdala subregional structure and intrinsic functional connectivity predicts individual differences in anxiety during early childhood. Biol Psychiatry 2014;75:892-900 CrossRef Medline

36. Sanz-Cortes M, Egaña-Ugrinovi G, Zupan R, et al. Brainstem and cerebellar differences and their association with neurobehavior in term small-for-gestational-age fetuses assessed by fetal MRI. Am J Obstet Gynecol 2014;210:452.e1-8 CrossRef Medline

37. Mürner-Lavanchy I, Steinlin M, Nelle M, et al. Delay of cortical thinning in very preterm born children. Early Hum Dev 2014;90:443-50 CrossRef Medline

38. Carver AR, Andrikopoulou M, Lei J, et al. Maternal pravastatin prevents altered fetal brain development in a preeclamptic CD-1 mouse model. PLoS One 2014;9:e100873 CrossRef Medline

39. Carver AR, Tamayo E, Perez-Polo JR, et al. The effect of maternal pravastatin therapy on adverse sensorimotor outcomes of the offspring in a murine model of preeclampsia. Int J Dev Neurosci 2014; 33:33-40 CrossRef Medline

40. Liu X, Zhao W, Liu H, et al. Developmental and functional brain impairment in offspring from preeclampsia-like rats. Mol Neurobiol 2015 Jan 10. [Epub ahead of print] CrossRef Medline

41. Hadjiiski LM, Tourassi GD, Galarreta-Valverde MA, et al. Characterization of vascular tree architecture using the Tokunaga taxonomy. In: Proceedings of the Annual Meeting of the SPIE Medical Imaging Conference, February 21-16. Orlando, Florida 